\title{
НЕЙРОКОРРЕКЦИОННЫЙ ПОТЕНЦИАЛ ЗАНЯТИЙ АДАПТИВНОЙ ФИЗИЧЕСКОЙ КУЛЬТУРЫ
}

\author{
Зайцева Лидия Владимировна \\ Полякова Анастасия Алексеевна \\ студенты
}

Научный руководитель: Шалина Ольга Сергеевна к.психол.н., доцент кафедры общей психологии ФГБОУ ВО «МГМСУ им. А.И. Евдокимова»

\begin{abstract}
Аннотация: B статье представлены результаты пилотажного исследования нейрокоррекционного потенциала занятий адаптивной физической культуры в системе реабилитации детей с нарушениями психического развития. Приведены результаты анализа динамики социальнокоммуникативных, нейропсихологических показателей и показателей психомоторного развития детей, принимавших участие в программе адаптивной физической культуры АНО «Школа героев».

Ключевые слова: нейрокоррекция, адаптивная физическая культура, эффективность АФК.
\end{abstract}

\section{NEUROCORRECTIONAL POTENTIAL OF ADAPTIVE PHYSICAL CULTURE CLASSES}

\section{Zaitseva Lidiya Vladimirovna Polyakova Anastasia Alekseevna Scientific adviser: Olga Sergeevna Shalina}

\begin{abstract}
A pilot study was conducted to determine the neurocorrective potential of adaptive physical culture classes and verify its effectiveness in the rehabilitation of children with mental development disorders. The dynamics of socio-communicative, neuropsychological indicators, as well as indicators of psychomotor development are presented and analyzed.
\end{abstract}

Keywords: neurocorrection, adaptive physical culture, efficiency of APC.

За последние 15 лет в России прирост детей с психическими нарушениями и расстройствами поведения составил 7 тысяч, а с нарушением 
центральной нервной системы (ЦНС) - 2,5 тысячи. У большинства из них отмечается несформированность различных высших психических функций (ВПФ). Данная работа поможет верифицировать эффективность занятий адаптивной физической культурой (АФК) в коррекции психомоторного развития детей, их когнитивных функций (памяти, мышления, внимания, пространственных представлений, речи), коммуникации и социальной адаптации [1].

Движение, моторика - это ведущая форма активности ребёнка, основа формирования психических процессов, физических и социальных навыков, развития в целом. В процессе специально организованной физической активности, занятий спортом, появляется дополнительная активация головного мозга за счет физиологических механизмов, что может способствовать улучшению его работы, а, следовательно, развитию ВПФ.

АФК - это комплекс мер спортивно-оздоровительного характера, направленных на реабилитацию и адаптацию к нормальной социальной среде людей с ограниченными возможностями, преодоление психологических барьеров, препятствующих ощущению полноценной жизни, а также сознанию необходимости своего личного вклада в социальное развитие общества [2, c. 7].

АФК, являясь одной из эффективных технологий абилитации детей с нарушениями психического развития (НПР) и наиболее естественной формой инклюзии, играет важную роль в реабилитации, поскольку гармонично включает в себя базовые принципы нейропсихологической коррекции. Коррекционно-развивающие техники АФК, опирающиеся на потенциал сенсорного и моторного опыта, играют особую роль в развитии памяти, мышления и воображения ребенка.

Нейропсихологический подход в настоящее время представляется актуальным направлением в работе не только с людьми, имеющими травму, но и с детьми с ограниченными возможностями здоровья (OB3), а также надёжным методологическим основанием для оценки эффективности программ АФК в абилитации детей с НПР.

В отличие от психометрического, нейропсихологический подход к диагностике делает акцент на самом процессе деятельности [3]. Благодаря ему возможно в полной мере оценить двигательную активность ребенка: общие характеристики двигательной сферы, предметно-бытовые и предметноигровые навыки, произвольную форму разных видов праксиса [4], а также 
выделенные Б.Г. Ананьевым аспекты психомоторных свойств: локомоции, мышечный тонус, особенности статического и динамического тремора, мышечное напряжение, точность движений рук и ног, графические движения [5].

\section{Общая информация об исследовании}

В 2020-2021 г. было проведено исследование динамики показателей психомоторного и психического развития детей с НПР, принявших участие в программах АФК «Адаптивный батут» и «Адаптивный скалодром», организованных АНО «Школа героев» на базе батутно-развлекательных парков «Небо» (Москва). В исследовании приняли участие 79 детей:

- Возраст респондентов - от 6 до 13 лет;

- 65 мальчиков (82\%) и14 девочек (18\%);

- У детей были установлены следующие диагнозы: детский аутизм (F-84.0), атипичный аутизм (F-84.1), врожденные аномалии века, слезного аппарата и глазницы (Q-90).

Задачи были сугубо прикладными - оценить эффективность этих программ АФК. Представлялось значимым выявить, какие аспекты психической сферы ребенка наиболее чувствительны к коррекции в процессе занятий. Это материал необходим для дальнейшей разработки принципов психологического сопровождения адаптивного спорта и определения его нейрокоррекционного потенциала.

Дети обследовались психологами и физиологами перед началом цикла занятий и по его завершению (через 2 месяца). Физиологическое исследование моторных функций включало оценку силы мышц рук, равновесия и баланса, координации движений, способности к дифференцированию параметров движения и моторную реакцию на сигнал.

Использовались стандартные и модифицированные нейропсихологические пробы:

1. Проба на соматогнозис направлена на исследование сформированности схемы тела;

2. Проба на слухоречевую память позволяет исследовать процессы восприятия и удержания слухоречевой информации;

3. Корректурная проба направлена на выявление утомляемости, оценки концентрации и устойчивости внимания;

4. Проба на оральный праксис позволяет исследовать кинестетическую организацию движений по речевой инструкции; 
5. Проба на зрительнаую память предназначена для определения объема кратковременной зрительной памяти;

6. Проба для оценки сформированности зрительно-пространственных представлений и зрительно-моторных координаций ребенка - рисунок (или копирование) трехмерного объекта (рисунок стола);

7. Графическая проба на динамический праксис позволяет исследовать возможность усвоения двигательной программы, плавность переключения с одного элемента программы на другой, автоматизацию двигательной серии;

8. Проба Хэда направлена на исследование возможности восприятия и воспроизведения нужного положения рук в пространстве, сформированности схемы тела;

9. Проба на зрительно-моторную координацию - методика «дорожки» (по Л.А. Венгеру) направлена на исследование внимания, контроля за собственными действиями, уровня развития точности движений рук;

10. Проба на воспроизведение и оценку ритмов предполагает исследование слухового восприятия и выполнения серийных движений;

11. Проба, направленная на исследование реципрокной координации движений, позволяет изучить сформированность механизмов серийной организации движений и межполушарного взаимодействия;

12. Проба на логико-грамматические конструкции направлена на изучение понимания пространственных и квазипространсвенных отношений.

13. Проба на распознавание эмоций [6].

14. Для интерференции использовалось задание на счёт.

\section{Полученные результаты}

Анализ значимости различий по критерию Т-Вилкоксона проведён в программе SPSS. Выбор критерия обусловлен типом данных (номинативные) и связь групп (выборки зависимые). Сравнивались результаты двух этапов диагностики - входного и итогового обследования (значимые различия при $\mathrm{p}$ $<0,05$ обозначены звёздочкой *).

Положительная динамика наблюдается по ряду критериев, однако малочисленность группы и сложность обследования детей, не вступающих в полноценный речевой контакт и крайне ограниченно понимающих и выполняющих инструкции, ограничили валидность результатов. Значимые различия выявлены по двум критериям - «Особенности вступления в контакт» и «Выраженность негативизма». 
Особенности вступления в контакт. При диагностике перед началом занятий на батуте в контакт вступали самостоятельно только $66 \%$, а по результатам диагностики после занятий вступали в контакт уже $84 \%$ детей. На первичном обследовании контакт был затруднен или неустойчив у $21 \%$, а совсем недоступен 13\%, на финальном же обследовании показатели по этим же критериям снизился - $13 \%$ и $3 \%$, соответственно (рис. 1 ).

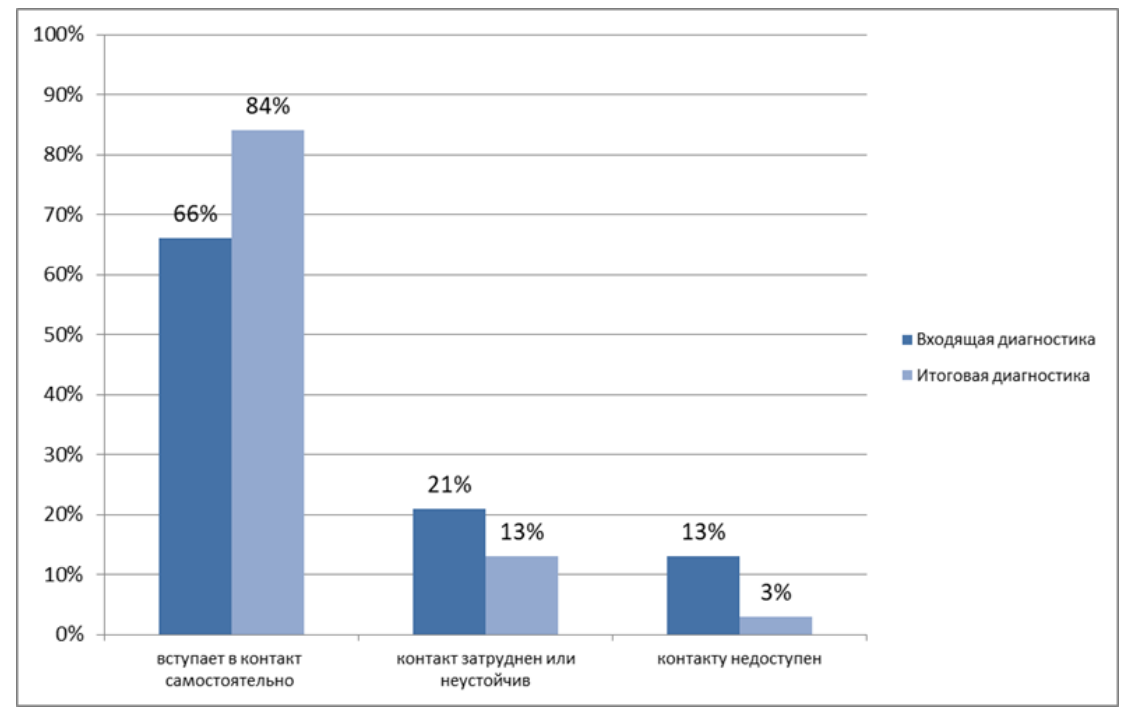

Рис. 1. Особенности вступления и поддержания контакта, \% от выборки *

Негативизм. Результаты обследования показали, что занятия спортом могут нивелировать негативные эмоциональные проявления ребенка, так на входящем обследовании $16 \%$ проявляли негативизм, по окончании программы адаптивного спорта показатель по критерию равняется 7\% (рис. 2).

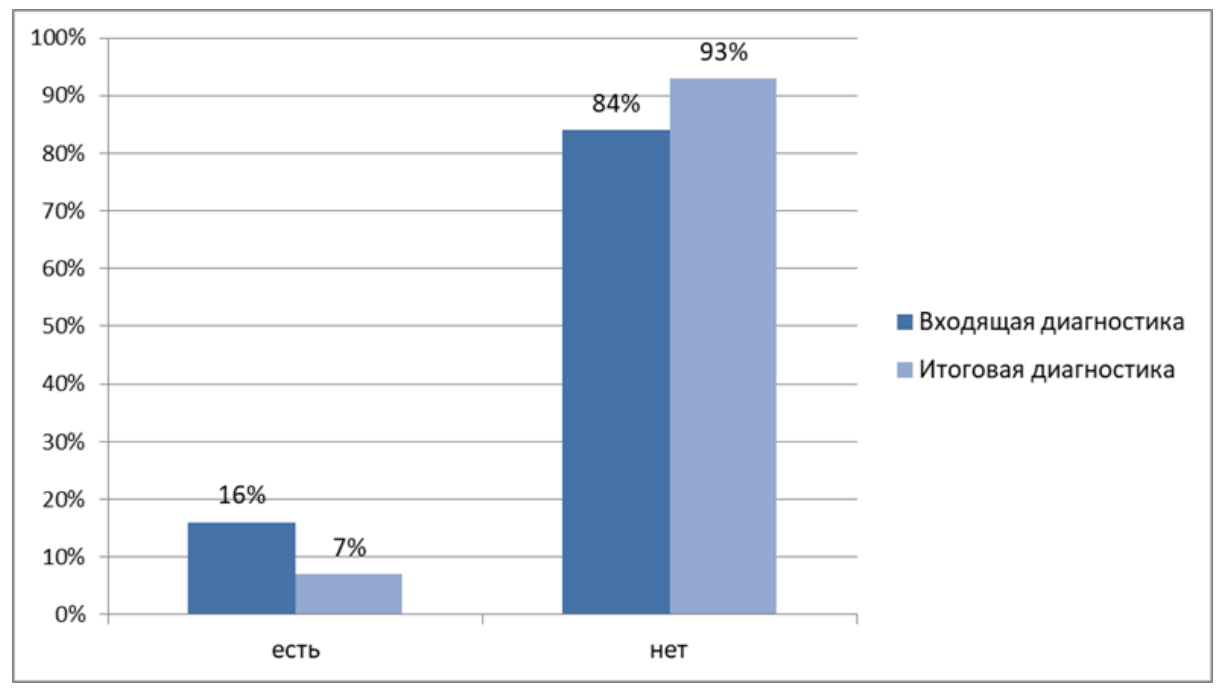

Рис. 2. Проявления негативизма, \% от выборки * 


\section{Реакции на инструкцию. Результаты по этому критерию} свидетельствуют о положительной динамике: перед началом программы 8\% детей не понимали инструкцию и неверно выполняли, а в конце только $1 \%$ также не понимали инструкцию (рис. 3).

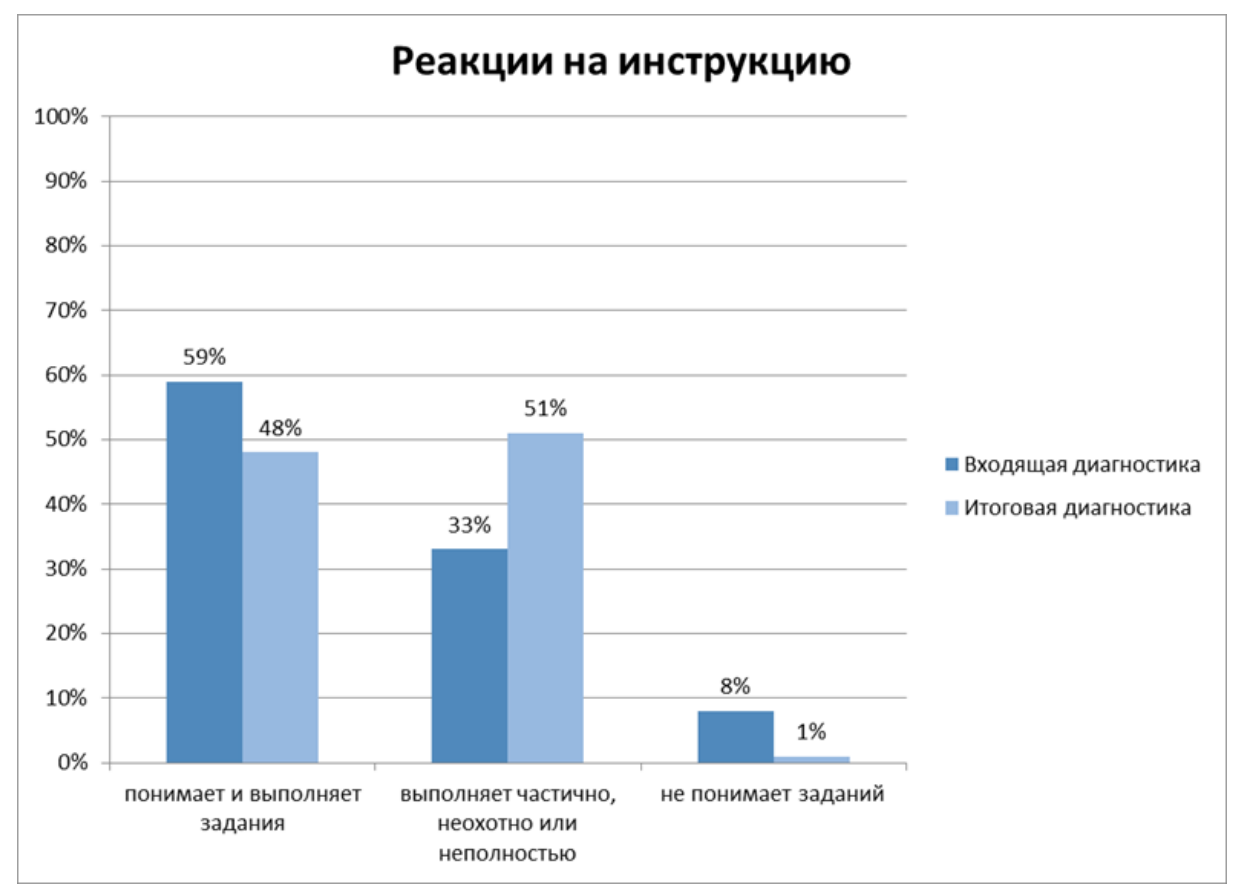

\section{Рис. 3. Реакции на инструкцию, \% от выборки}

Инертность. При первом обследовании этот показатель был значительно выражен у $26 \%$ испытуемых, а при втором обследовании сильная выраженность снизилась инертности наблюдалась у $10 \%$ (рис. 4).

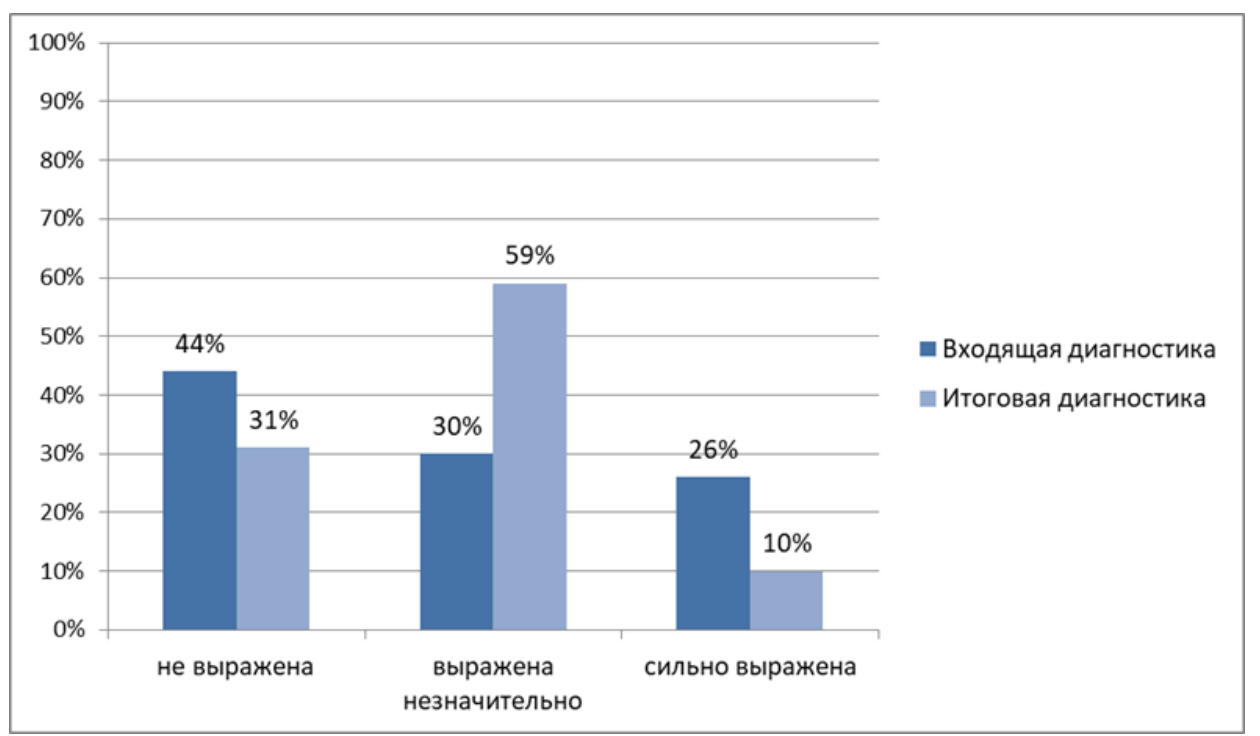

Рис. 4. Инертность, \% от выборки 
Импульсивность. На начальном этапе обследования импульсивность была сильно выражена у 21\% испытуемых и снизилась до 6\% при повторном обследовании (рис. 5).

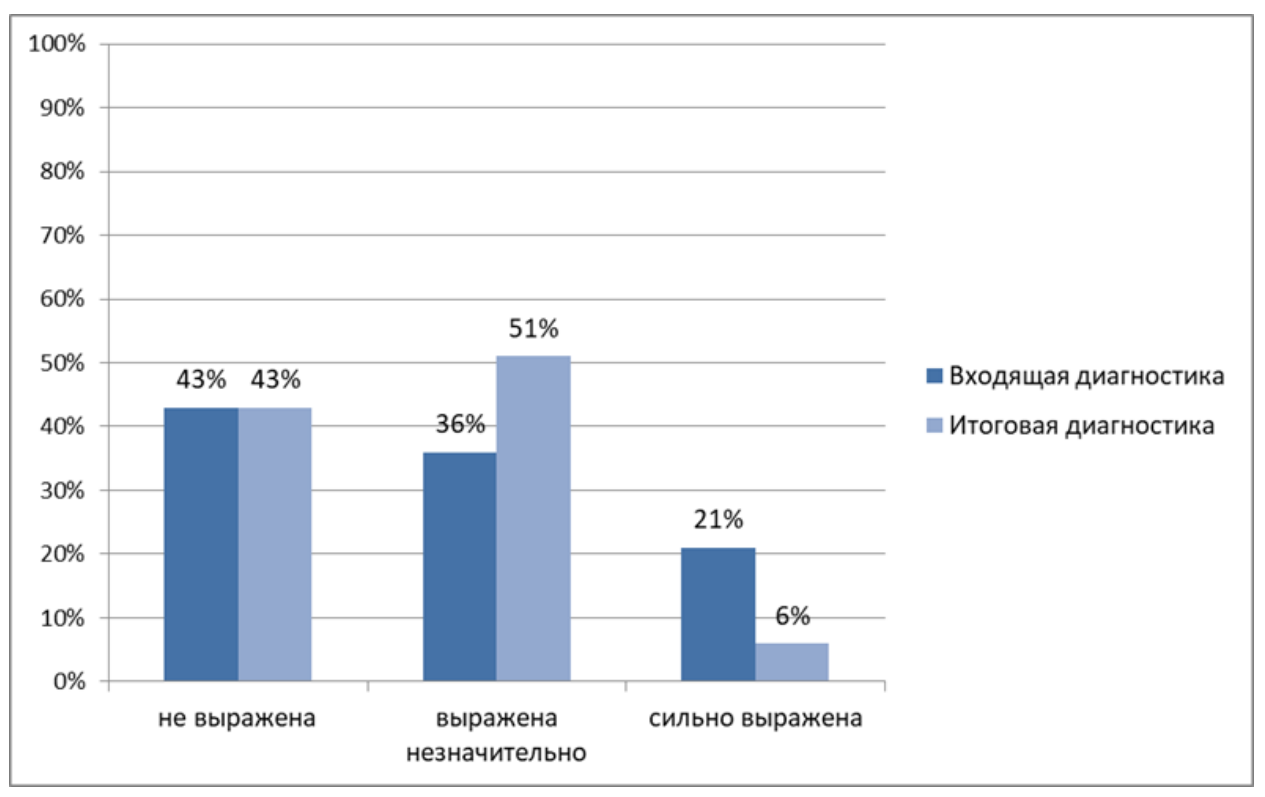

Рис. 5. Импульсивность, \% от выборки

Сформированность моторной сферы. На входном обследовании уровень сформированности моторной сферы был в пределах возрастной нормы только у $19 \%$ детей, а на итоговым - у 21\%; грубое отставание моторной сферы на входящем обследовании наблюдалась у $21 \%$, а на итоговом - у $12 \%$ (рис. 6).

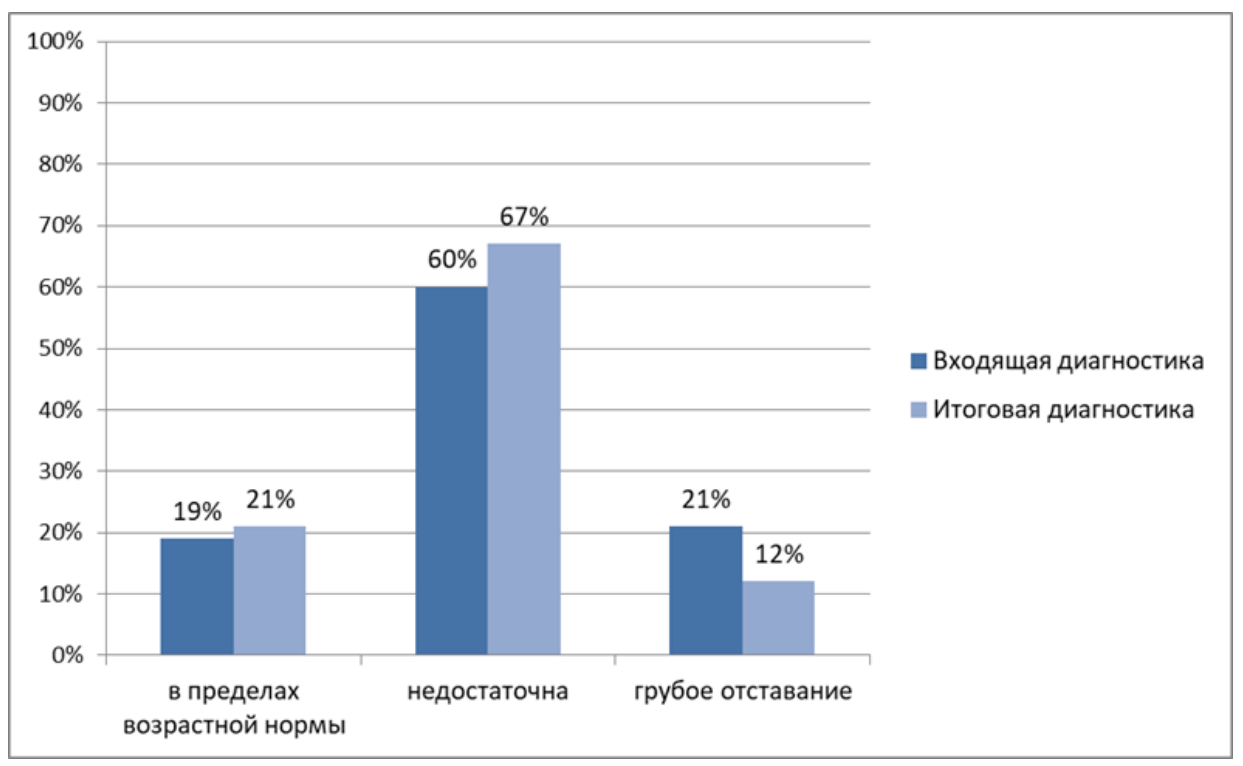

Рис. 6. Сформированность моторной сферы, \% от выборки 


\section{Обсуждение результатов}

В начале программы, когда была сформирована группа, дети характеризовались неуверенностью, слабостью и недостаточностью контакта и коммуникации, склонностью к игнорированию или невыполнению инструкций, более или менее выраженными инертностью, импульсивностью. У всех детей была выражена недостаточность нейродинамического компонента деятельности и снижена потребность во внимании и встречном интересе. Речь у большинства детей задержана, не сформирована по возрасту или отсутствует. Критичность, произвольное внимание, пространственные представления и мышление сформированы ниже возрастной нормы, недостаточны. Моторная сфера также отличалась незрелостью, слабостью, недостаточностью.

На период завершения коррекционной программы дети продемонстрировали положительную динамику по ряду параметров. Их внимание и готовность следовать инструкциям усилились, дети активнее стали следить за собеседником, чаще откликаться на задание, проявлять большую дисциплинированность. Что особенно важно, больше детей стали ориентироваться на общение, проявлять интерес к людям, удерживать контакт.

В их поведении меньше прослеживалась импульсивность и, соответственно, уровень произвольного внимания несколько повысился (хотя всё же для организации своего поведения им недостаточно собственных стремлений, дети нуждаются во внешних инструкциях и контроле).

Отмечался более высокий уровень общего моторного развития, дети стали более уверенно совершать движения, легче формировать программы действий. Инертность и склонность к повторяющимся стереотипным действиям снизились.

Интересно, что наметилась и некоторая тенденция к увеличению доли детей, опирающихся на наглядно-действенное мышление, а также демонстрирующих и более сложные приёмы мышления. Улучшились конструктивные способности детей.

\section{Выводы}

Конечно, сложная структура дефекта психического развития и неравномерность развития каждого ребёнка, а также глубинная органическая причина нарушения развития не могут быть полностью скомпенсированы и восполнены краткосрочной программой тренировок. Однако наблюдаемая 
динамика позволяет с уверенностью говорить о возможности коррекции базовых уровней развития. Дети, участвующие в программе адаптивного спорта, демонстрируют готовность обучаться, развитие активного внимания и интереса к тренеру / педагогу, снижение импульсивности и инертности, осложняющих любое коррекционно-развивающий процесс. Полученный опыт активности благоприятно сказался и на развитии мышления: дети в большей степени овладели конструктивными навыками, начали демонстрировать более сложные приёмы мышления.

По результатам пилотажного исследования, программы АФК представляются эффективной техникой абилитации детей с НПР, однако значимо улучшаются скорее социально-коммуникативные показатели; собственно нейропсихологические и показатели психомоторного развития требуют более продолжительной коррекции.

Адаптивный спорт способствует развитию и коррекции высших психических функций, разработка программ адаптивного спорта и его внедрение в нейрокоррекционное направление необходимо для увеличения эффективности нейрокоррекции.

\section{Список литературы}

8. Евсеев С.П. Теория и организация адаптивной физической культуры: учебник/С.П. Евсеев. - Москва: Спорт, 2016. - 616 с.

9. Дмитриев А.А. Адаптивная физическая культура в специальном образовании. - Москва: Академия, 2002. - 176 с.

10. Глозман Ж.М. Нейропсихологическое обследование: качественная и количественная оценка данных. - М.: Смысл, 2012. - 264 с.

11. Цветкова Л.С. Методика нейропсихологической диагностики детей. Изд. 2-е, исправленное и дополненное. - М.: «Российское педагогическое агентство», «Когито-центр», 1998. - 128 с.

12. Ананьев Б.Г. Человек как предмет познания - СПб.: Питер, 2001. 288 c.

13. Ахутина Т.В. Методы нейропсихологического обследования детей 69 лет. - М.: Изд-во В. Секачев, 2016. - 280 с.

() Л.В. Зайцева, А.А. Полякова, 2021 